\title{
Effects of alloying elements and temperature on the elastic properties of W-based alloys by first-principles calculations
}

Yong-Jie Hu ${ }^{1 *}$, Shun-Li Shang ${ }^{1}$, Yi Wang ${ }^{1}$, Kristopher A. Darling ${ }^{2}$, Brady G. Butler ${ }^{2}$, Laszlo J. Kecskes $^{2}$, and Zi-Kui Liu ${ }^{1}$

${ }^{1}$ Department of Materials Science and Engineering, The Pennsylvania State University, University Park, PA 16802, USA

${ }^{2}$ U.S. Army Research Laboratory, Weapons and Materials Research Directorate, RDRL-WMMF, Aberdeen Proving Ground, MD 21005, USA

*Corresponding author:

Yong-Jie $\mathrm{Hu}$

yoh5120@psu.edu 


\begin{abstract}
The influence of various transition alloying elements (X's) on the elastic properties of Wbased alloys has been studied via first-principles calculations on the basis of density functional theory. Here, nineteen transition metal alloying elements $(\mathrm{X})$ are considered: Ti, V, Cr, Fe, Co, $\mathrm{Ni}, \mathrm{Y}, \mathrm{Zr}, \mathrm{Nb}, \mathrm{Mo}, \mathrm{Ru}, \mathrm{Rh}, \mathrm{Pd}, \mathrm{Hf}, \mathrm{Ta}, \mathrm{Re}, \mathrm{Os}, \mathrm{Ir}$, and Pt. It is found that (i) the bulk modulus of the dilute $\mathrm{W}-\mathrm{X}$ alloy decreases with increasing its equilibrium volume, particularly, for the alloying elements in the same period; (ii) all of the alloying elements decrease the shear modulus of $\mathrm{BCC} \mathrm{W}$; and (iii) the largest decrease of elastic properties of $\mathrm{W}$ is due to alloying element $\mathrm{Y}$. In addition, it is shown that the changes of elastic properties of $\mathrm{W}$ caused by the alloying elements are traceable from the electron charge density distribution, resulting in a bonding distortion between $\mathrm{W}$ and the alloying atoms. Using the quasi-static approach based on the Debye model, the elastic properties of these $\mathrm{W}-\mathrm{X}$ alloys at finite temperatures are predicted. Calculated properties of BCC $\mathrm{W}$ and the $\mathrm{W}-\mathrm{X}$ alloys are in favorable agreement with available experimental measurements.
\end{abstract}

\title{
Keywords:
}

First-principles calculations; Tungsten-based alloys; Elastic properties; Quasi-static approach; Electronic structure 


\section{Introduction}

W-based alloys have high melting temperatures, high density, high strength and good resistance to gamma and neutron radiations [1]. Currently, in order to further increase its toughness and ductility, similar to that used in Ni-based superalloys [2], the primary alloying element for BCC W has been Re $[1,3,4]$. However, Re is one of the least abundant elements in the Earth's crust, resulting in its extremely high price [2]. In response to this dilemma, there is significant on-going research to identify alternative alloying elements for the development of Wbased alloys with similar or improved mechanical properties. However, it is likely that the use of other not-yet-tried alloying additions would lead to more complex compositions. Therefore, it is desirable to gain a fundamental understanding of systematic alloying effects on the mechanical properties of $\mathrm{W}$, as a function of temperature. In the present work, we select elasticity for study, as it is hypothesized that the early stage of deformation which reflects the material's capability to recover its original shape after mechanical loading. In addition, the elasticity of single crystals and the associated polycrystalline aggregate properties can provide information about a vast number of material-related behaviors, such as mechanical anisotropy and stability [5], Debye temperature [6], hardness [7], melting temperatures [8], ductility/brittleness [9], and creep rate [10].

Although first-principles approaches based on density functional theory have been proven to be a reliable and efficient method in predicting elastic properties of various alloying systems $[5,8,11-13]$, little work has been performed on the elastic properties of $\mathrm{W}$ and its alloys in a comprehensive manner, especially at finite temperatures. Through first-principles calculations, the present work aims to close this gap in understanding by investigating systematically the effects of alloying elements (X's) on the elastic properties of $\mathrm{BCC} \mathrm{W}$, as a function of 
temperature, including isothermal and isentropic elastic stiffness components, bulk modulus, shear moduli, Young's modulus, and elastic anisotropy. Nineteen transition metal alloying elements are considered: Ti, V, Cr, Fe, Co, Ni, Y, Zr, Nb, Mo, Ru, Rh, Pd, Hf, Ta, Re, Os, Ir, and Pt. In addition, the trends, regularities of the predicted elastic properties, and their electronic origins are discussed.

\section{Computational Methodology}

\subsection{First-Principles Elastic Properties at 0 K}

First-principles elastic stiffness constants of a series of $\mathrm{W}-1.85$ at.\% $\mathrm{X}$ solid solutions are calculated by using the Vienna Ab-initio Simulation Package (VASP) [14]. A bcc $3 \times 3 \times 3$ supercell comprised of $53 \mathrm{~W}$ atoms and one additional atom associated with alloying element $\mathrm{X}$ is used. Further, the projector augmented wave method (PAW) [15] and the exchange-correlation functional depicted by the generalized gradient approximation (GGA-PW91) are employed [16]. The energy cutoff of the plane wave is set as $350 \mathrm{eV}$. For all the calculations, the supercells are relaxed by the Methfessel-Paxton method [17]. The Brillouin-zone integration is performed by using a tetrahedron method with Blöchl corrections [18] and a Monkhorst-Pack $k$-points grid of $8 \times 8 \times 8$ [17]. The energy convergence criterion of the electronic self-consistency is set as $10^{-6}$ eV/atom for all of the calculations. The contour plots of the differential charge density are generated using VESTA [19,20].

Elastic stiffness constants in the present work are calculated based on the efficient stressstrain method [21]. Specifically, independent sets of strains are applied to deform the supercell in the elastic range. For a given set of strains, $\varepsilon_{i}=\left(\varepsilon_{i, 1}, \varepsilon_{i, 2} \cdots, \varepsilon_{i, 6}\right)$, lattice vectors of the strained supercell, R', in Cartesian coordinates can be calculated as, 
Equation 1

$$
\mathbf{R}^{\prime}=\mathbf{D R}=\left(\begin{array}{ccc}
1+\varepsilon_{i, 1} & \varepsilon_{i, 6} / 2 & \varepsilon_{i, 5} / 2 \\
\varepsilon_{i, 6} / 2 & 1+\varepsilon_{i, 2} & \varepsilon_{i, 4} / 2 \\
\varepsilon_{i, 5} / 2 & \varepsilon_{i, 4} / 2 & 1+\varepsilon_{i, 3}
\end{array}\right)\left(\begin{array}{ccc}
a_{1} & a_{2} & a_{3} \\
b_{1} & b_{2} & b_{3} \\
c_{1} & c_{2} & c_{3}
\end{array}\right)
$$

where $\mathbf{R}$ are the lattice vectors of the unstrained supercell. The stresses in response to the given strains are calculated via the aforementioned first-principles approach.

Generally, in order to calculate the complete elastic stiffness constants, six independent sets of strains are applied on the structure:

\section{Equation 2}

$$
\left(\begin{array}{lll}
\varepsilon_{1,1} & & \varepsilon_{1,6} \\
\varepsilon_{2,1} & & \varepsilon_{2,6} \\
\varepsilon_{3,1} & & \varepsilon_{3,6} \\
\varepsilon_{4,1} & & \varepsilon_{4,6} \\
\varepsilon_{5,1} & & \varepsilon_{5,6} \\
\varepsilon_{6,1} & & \varepsilon_{6,6}
\end{array}\right)=\left(\begin{array}{llllll}
x & & & & & \\
& x & & & & \\
& & x & & & \\
& & x & & \\
& & & x & \\
& & & & x
\end{array}\right)
$$

where $x= \pm 0.01$ in the present work, and the strain components not shown are zero. Using the six sets of corresponding stresses from first-principles, the elastic stiffness constants at a fixed volume, $c_{i j}(V)$, can be determined based on Hooke's law as follows:

\section{Equation 3}

$$
\left(\begin{array}{llllll}
c_{11} & c_{12} & c_{13} & c_{14} & c_{15} & c_{16} \\
c_{21} & c_{22} & c_{23} & c_{24} & c_{25} & c_{26} \\
c_{31} & c_{32} & c_{33} & c_{34} & c_{35} & c_{36} \\
c_{41} & c_{42} & c_{43} & c_{44} & c_{45} & c_{46} \\
c_{51} & c_{52} & c_{53} & c_{54} & c_{55} & c_{56} \\
c_{61} & c_{62} & c_{63} & c_{64} & c_{65} & c_{66}
\end{array}\right)=\left(\begin{array}{lll}
\sigma_{1,1} & & \sigma_{1,6} \\
\sigma_{2,1} & & \sigma_{2,6} \\
\sigma_{3,1} & \ldots & \sigma_{3,6} \\
\sigma_{4,1} & & \sigma_{4,6} \\
\sigma_{5,1} & & \sigma_{5,6} \\
\sigma_{6,1} & & \sigma_{6,6}
\end{array}\right)\left(\begin{array}{lll}
\varepsilon_{1,1} & & \varepsilon_{1,6} \\
\varepsilon_{2,1} & & \varepsilon_{2,6} \\
\varepsilon_{3,1} & \ldots & \varepsilon_{3,6} \\
\varepsilon_{4,1} & & \varepsilon_{4,6} \\
\varepsilon_{5,1} & & \varepsilon_{5,6} \\
\varepsilon_{6,1} & & \varepsilon_{6,6}
\end{array}\right)^{-1}
$$

For cubic crystals, there are only three independent elastic stiffness constants, $c_{11}, c_{12}$, and $c_{44}$. Therefore, instead of six, only two linearly independent sets of strains are needed to calculate all the cubic elastic stiffness constants. In the present work, $c_{11}$ and $c_{12}$ are obtained from the set of strains, $\varepsilon_{1}$, and $c_{44}$ is obtained from the set of strains, $\varepsilon_{4}$. 
Based on the single crystal elastic stiffness constants obtained, aggregate properties of the bulk $(B)$, shear $(G)$, and Young's $(E)$ moduli and Poisson's ratio $(v)$ associated with polycrystals are estimated by means of the Voigt approximation [22]; this provides the upper bound of elastic properties in terms of uniform strains. For a cubic crystal,

$\begin{array}{ll}\text { Equation } 4 & B=\left(c_{11}+2 c_{12}\right) / 3 \\ \text { Equation 5 } & G=\left(c_{11}-c_{12}+3 c_{44}\right) / 5 \\ \text { Equation 6 } & E=9 B G /(3 B+G) \\ \text { Equation } 7 & v=(3 B-2 G) / 2(3 B+G)\end{array}$

The shear modulus along the closest-packed $\langle 111\rangle$ direction is also calculated since this is the shearing direction for dislocation glide in BCC W [1]. For cubic crystals [23],

Equation 8

$$
G_{111}=\frac{3 c_{44}\left(c_{11}-c_{12}\right)}{c_{11}-c_{12}+4 c_{44}}
$$

The universal elastic anisotropy index, $A^{U}$, can be calculated based on the Zener anisotropy ratio, $A^{Z}$. For a cubic crystal [11],

Equation 9

$$
A^{U}=\frac{6}{5}\left(\sqrt{A^{Z}}-\frac{1}{\sqrt{A^{Z}}}\right)^{2}
$$

where $A^{Z}=2 c_{44} /\left(c_{11}-c_{12}\right)[5] . A^{U}=0$ or $A^{Z}=1$ indicates an isotropic crystal.

\subsection{Thermodynamic and Elastic Properties at Finite Temperatures}


The elastic stiffness constants at finite temperatures could be estimated by a quasi-static approach in terms of the predicted Helmholtz free energy at finite temperatures and the predicted elasticity as a function of volume at $0 \mathrm{~K}$ [24]. The Helmholtz free energy, $F(V, T)$, at volume $V$ and temperature $T$ can be calculated using the quasi-harmonic approach [25],

$$
\text { Equation } 10 \quad F(V, T)=E(V)+F_{\text {ele }}(V, T)+F_{v i b}(V, T)
$$

The first part, $E(V)$, is a static energy at $0 \mathrm{~K}$ and, without the contribution of the zero-point vibration, it is obtained by a fitting of the first-principles energy vs. volume data points based on a four-parameter Birch-Murnaghan equation of state (EOS) $[5,25]$,

$$
\text { Equation } 11 \quad E(V)=a+b V^{-2 / 3}+c V^{-4 / 3}+d V^{-2}
$$

where $\mathrm{a}, \mathrm{b}, \mathrm{c}$ and $\mathrm{d}$ are fitting parameters from which the equilibrium energy $\left(E_{0}\right)$, volume $\left(V_{0}\right)$ bulk modulus $\left(B_{0}\right)$, and the first derivative of bulk modulus with respect to pressure ( $\left.B_{0}^{\prime}\right)$ can be estimated. In the present work, six data points are generally used for the EOS fitting for each alloy. The second part in the Equation $10, F_{\text {ele }}(V, T)$, represents the thermal electronic contribution which is calculated from the electronic density of states (DOS) obtained from firstprinciples calculations $[25,26]$. This part is necessary due to the non-zero electronic density at the Fermi level associated with metals. The third part, $F_{v i b}(V, T)$, is the contribution of lattice vibrations to the Helmholtz free energy. In the present work, for sake of simplicity and efficiency, the vibrational contribution is depicted based on the Debye-Grüneisen model [25],

$$
\text { Equation } 12 \quad F_{v i b}(V, T)=\frac{9}{8} k_{B} \Theta_{D}+k_{B} T\left\{3 \ln \left[1-\exp \left(-\frac{\Theta_{D}}{T}\right)\right]-D\left(\frac{\Theta_{D}}{T}\right)\right\}
$$

where $k_{B}$ is the Boltzmann constant and $D\left(\Theta_{D} / T\right)$ the Debye function. The Debye temperature $\Theta_{D}$ can be calculated by, 
Equation 13

$$
\Theta_{D}=s\left(6 \pi^{2}\right)^{\frac{1}{3}} \frac{\hbar}{k_{B}} V_{0}^{\frac{1}{6}}\left(B_{0} / M\right)^{\frac{1}{2}}\left(V / V_{0}\right)^{\gamma}
$$

where $\mathrm{M}$ is the atomic mass and $s$ a scaling factor which is calculated based on the Poisson's ratio of pure $\mathrm{W}[6]$. The Grüneisen constant, $\gamma$, is defined as $\gamma=\left[\left(1+B_{0}^{\prime}\right) / 2-x\right]$, with $x=2 / 3$ due to the high melting temperature of $\mathrm{W}[25]$.

As temperature and volume are two independent variables of the Helmholtz free energy, the equilibrium volume at temperatures of interests, $V(T)$, can be directly calculated from Equation 10. Combining this with the predicted elastic constants $c_{i j}(V)$, the isothermal elastic stiffness constants at finite temperatures can be estimated as $c_{i j}(T)=c_{i j}(V(T))[11,24]$. It should be mentioned that this approach assumes that the change of elastic stiffness mainly depends on the volume expansion and anharmonicity effects and contributions due to the fluctuation of microscopic stress tensors are not taken into consideration.

Generally, measured elastic constants obtained via the resonance method are isentropic because the elastic waves travel faster than the rate of heat conduction, leading to the measurements being adiabatic in nature. Therefore, in order to compare them with experiments, the calculated isothermal elastic stiffness constants need to be converted to the isentropic ones. For cubic crystals, the thermodynamic relationships between the isothermal $c_{i j}^{T}$ and isentropic $c_{i j}^{S}$ are as follows [27],

Equation $14 \quad c_{11}^{S} / c_{11}^{T}=c_{12}^{S} / c_{12}^{T}=C_{P} / C_{V}$ and $c_{44}^{S}=c_{44}^{T}$

where $C_{P}$ and $C_{V}$ are heat capacities at constant pressure and constant volume, respectively, which can be estimated from Helmholtz free energy given in Equation 10[25]. 


\section{Results and Discussion}

In this section, we first report the regularities of elastic properties and their electronic origins for the specific W-X alloys at $0 \mathrm{~K}$ (Sec. 3.1). Second, we present the elasticity of the W-X alloys at finite temperatures (Sec. 3.2).

\subsection{Elastic Properties at 0 K}

Table 1 summarizes the calculated elastic properties of the $\mathrm{W}-\mathrm{X}$ alloys at $0 \mathrm{~K}$ as well as the equilibrium volumes and lattice parameters predicted by the EOS fitting. It is found that the present prediction of equilibrium volume of $\mathrm{BCC} \mathrm{W}$ agrees well with experimental data and previous calculations [5,28]. For the elastic stiffness components, the present $c_{11}$ and $c_{12}$ values are in good agreement with the experimental data at $4.2 \mathrm{~K}$ [28], with the largest difference less than $6 \%$ for $c_{12}$. The calculated $c_{44}$ value in the present work has a $12 \%$ deviation compared to the experimental data, but it agrees well with a previous calculation [5].

To investigate the lattice distortion induced by the solute atoms, Figure 1 plots the EOS predicted equilibrium volumes from Table 1 compared to the values calculated based on Vegard's law for pure elements with a BCC structure [5]. It is shown that the effect of X on the volume of the dilute $\mathrm{W}-\mathrm{X}$ alloys can be represented as a mechanical intermixing for the alloying elements which have atomic volumes close to BCC W. Deviation between the first-principles calculation and the prediction from Vegard's law is found when the alloying element has a large disparity of its atomic volume compared to that of BCC W. On one hand, smaller alloying elements lead to positive deviations, such as those of $\mathrm{Ni}$ and $\mathrm{Co}$. On the other hand, large negative deviations are found for alloying elements $\mathrm{Y}, \mathrm{Zr}$, and $\mathrm{Hf}$, which all have much larger atomic volumes than BCC W. The large deviations from Vegard's law indicate that strong 
interactions exist between these solute elements and $\mathrm{W}$, in addition to the mechanical mixing between them. Such interactions can be ascribed to the change of the local electronic environment of the solute atom when it is substituted for a W atom in the BCC W matrix [29].

For dilute solid solutions, the changes of lattice parameter can be approximated as a linear function of alloying concentration [30],

Equation 15

$$
a_{W_{53} X}=a_{W_{54}}+k_{X} x_{X}
$$

where $a_{W_{53} X}$ and $a_{W_{54}}$ are the lattice parameters of the $\mathrm{W}-\mathrm{X}$ solutions and pure $\mathrm{W}$, respectively, $x_{X}$ is the mole fraction of alloying elements, and $k_{X}$ is defined as a linear regression coefficient for element X. Calculated lattice parameters of the W-X solutions and the corresponding linear regression coefficients are listed in Table 1. Figure 2 plots the linear regression coefficients vs. the lattice parameters of alloying elements with the BCC crystal structure. Available experimental values, obtained by a fitting of experimental lattice parameters [31,32], are also included, showing a good agreement. It is found that the linear regression coefficients are generally proportional to the lattice parameters of the alloying elements in the BCC structure. However, Ti and Y are slightly off the observed trend.

To illustrate the effect of alloying elements on the elastic modulus of BCC W, Figure 3 displays the calculated bulk moduli and compares them to those obtained from a linear combination of the pure elemental moduli in the $\mathrm{BCC}$ structure in terms of their mole fractions. For most alloying elements studied, it is found that the bulk modulus of the W-X dilute alloy can be approximated as a mechanical mixing of the bulk moduli of $\mathrm{X}$ and $\mathrm{W}$. However, similar to the volume analysis described in Figure 1, deviations from this trend are observed for $\mathrm{Y}, \mathrm{Zr}$, and Hf, scaling with their respective atomic numbers, which, by the way, all have the HCP structure and 
possess fewer valence electrons than W. However, it is noted that no clear correlation is found between the shear moduli of the $\mathrm{W}-\mathrm{X}$ dilute alloys and those of the pure alloying elements with a BCC structure.

Previously, it has been shown that elastic moduli are generally correlated to equilibrium volume $[5,11,12]$. Therefore, the calculated polycrystalline bulk and shear moduli of the $\mathrm{W}_{53} \mathrm{X}$ alloys are plotted as a function of the equilibrium volume of alloys in Figure $4 \mathrm{a}$ and $4 \mathrm{~b}$. As seen in Figure 4a, the alloys with smaller equilibrium volumes have larger bulk moduli. In addition, within each group of $3 \mathrm{~d}, 4 \mathrm{~d}$, and $5 \mathrm{~d}$ alloying elements, it is found that the bulk modulus of $\mathrm{W}_{53} \mathrm{X}$ decreases linearly with increasing equilibrium volume. Such trend was also reported for Ni- and Mg-based alloys [11,12]. Other than that for the bulk modulus, no clear correlation can be found between shear modulus and equilibrium volume, as is shown in Figure 4b. It is observed that all of the alloying elements decrease the shear modulus of BCC W to some extent. More precisely, the alloying effects on the shear modulus are found to correlate with the number of the valence electrons of the alloying elements, as shown in Figure 5. For the alloying elements of $\mathrm{Cr}$ and $\mathrm{Mo}$ with the same numbers of valence electrons as $\mathrm{W}$, the reductions of shear modulus are not significant compared to the other alloying elements. For the elements containing 3 and 5 electrons in their d orbital, respectively, such as $\mathrm{V}, \mathrm{Nb}, \mathrm{Ta}, \mathrm{Fe}, \mathrm{Ru}$, and $\mathrm{Os}$, the effect of alloying on shear modulus is moderate. As the disparity of the number of the valence electrons between the alloying element and $\mathrm{W}$ becomes larger, the shear modulus of BCC $\mathrm{W}$ deceases further. For example, there is a significant decrease of the shear modulus of BCC W alloying with $\mathrm{Y}, \mathrm{Zr}$, and Pd. A similar trend is also observed for the shear modulus along the $\langle 111\rangle$ direction $\left(G_{111}\right)$. Since valence electrons are crucial to the formation of chemical bonds, this trend implies that 
alloying effects on the shear modulus of $\mathrm{W}$ can be understood in terms of the interatomic bonds between the W and the solute atoms. Detailed discussion is provided in a later paragraph.

The elastic deformation behavior of crystalline materials originates from the stretching of atomic bonds when a force is applied. For example, the bulk modulus, which represents the compressibility of a crystal under hydrostatic pressure, is generally related to the stiffness of the chemical bonds. Therefore, it is interesting and important to investigate the electronic origins further, more particularly, how the elastic properties of the $\mathrm{W}-\mathrm{X}$ alloys are changing. According to Miedema et al. and $\mathrm{Li}$ and $\mathrm{Wu}$, (see references $[33,34]$ ), for pure alkali metals and nontransition metals, $\sqrt{B / V_{m}}$ is linearly proportional to the electron density at the boundary of the Wigner-Seitz cell, where $B$ is the bulk modulus and $V_{m}$ is the molar volume of the element. The electron density at the boundary of the Wigner-Seitz cell, $n$, can be calculated via $n=Z_{B} / V$, where $Z_{B}$ is the bond valence of the element and $V$ is the atomic equilibrium volume at $0 \mathrm{~K}$. This linear relationship has been validated for Ni-based alloys by taking into account the effect of alloying elements on $n$ [8]. Here, in a similar manner, we compute $n$ for $\mathrm{W}_{53} \mathrm{X}$ based on a linear combination of the constituent elements, similar to Vegard's law,

$$
\text { Equation } 16 \quad n_{W_{53} X}=\left(\frac{53}{54} n_{W} V_{W}+\frac{1}{54} n_{X} V_{X}\right) / V_{W_{53} X}
$$

where the electron densities, $n_{W}$ and $n_{X}$, are calculated from equilibrium volume[5] and bond valence data[34] of the pure elements found in published literature. Figure 6 shows that a linear relationship between $\sqrt{B / V_{m}}$ and electron density $n$ is obeyed in dilute W-based alloys. It is found that the bulk modulus of $\mathrm{W}_{53} \mathrm{X}$ is positively proportional to the electron density, $n_{W_{53} X}$. In fact, the value of $n$ reflects how dense the electron gas of a metallic system is. This is because 
the bond valence $\left(Z_{B}\right)$ is defined as the number of participating electrons where each atom contributes to the electron gas[35]. Thus, a higher electron density $n$ indicates a denser electron gas, implying stiffer chemical bonds between the atoms[36]. Therefore, the $\mathrm{W}_{53} \mathrm{X}$ alloys with a higher electron density, $n$, are anticipated to have stiffer chemical bonds between atoms, which, in turn, would result in a larger bulk modulus.

The influence of alloying elements on the shear modulus along the [111] direction of $\mathrm{W}$ can be better understood in terms of the differential charge densities that can be represented by the charge density difference for the calculated structure with and without the self-consistent calculations. Figure $7 \mathrm{a}, 7 \mathrm{~b}, 7 \mathrm{c}$, and $7 \mathrm{~d}$ show the differential charge density plots in the (11̄0)plane of $\mathrm{W}_{53} \mathrm{X}$ (with $\mathrm{X}=\mathrm{W}, \mathrm{Mo}, \mathrm{Ti}$, and $\mathrm{Pd}$ ) revealing the bonding environments between nearest neighbors. The relative intensity and directionality of the differential charge density are illustrated by a combination of color mapping and contour plotting. As seen from Figure 7a, the charge density of pure $\mathrm{W}$ is higher between adjacent atoms in the (110) plane along the <111> directions; note, this distance (dashed arrow in Figure 7a) is the shortest distance between two W atoms. This implies that the interatomic bonds along the $<111>$ directions in pure $\mathrm{W}$ are stronger than any other directions. As a result, the shear modulus along the $<111\rangle$ directions are expected to be larger than that associated with a polycrystalline microstructure (Table 1). Instead, from a polycrystalline microstructure, an average of shear moduli sampled from all directions would be expected. Furthermore, as seen from Figure $7 \mathrm{c}$ and $7 \mathrm{~d}$, the W-X bonds for W-Ti and W-Pd along the $<111>$ directions are distorted and weaker than the W-W bonds. Correspondingly, the shear moduli $G_{111}$ of $\mathrm{W}_{53} \mathrm{Ti}$ and $\mathrm{W}_{53} \mathrm{Pd}$ are much lower than that of pure $\mathrm{W}$ (Table 1). By analogy, the change of $G_{111}$ caused by the alloying element Mo is relatively small; this can be attributed to the 
fact that W-Mo bonds are very similar in nature to W-W type bonds (Figure 7b). Note, the crystal structures of Mo and $\mathrm{W}$ are isomorphous and they have similar atomic radii.

The observed decrease of the elastic shear moduli can be interpreted as a form of lattice softening induced by the alloying elements. Usually, it is considered that lattice softened alloys would lead to and may exhibit good plasticity due to the change of elasticity and crystal stability $[23,37,38]$. For example, the ideal shear strength in BCC crystals is governed by the shear modulus along the $<111>$ directions, $G_{111}$ [23]. As $G_{111}$ decreases, the crystal lattice is expected to be softer or more deformable in response to a shear stress. Based on the Peierls-Nabarro model, the size of the dislocation glide barrier is also proportional to the shear modulus along the Burger's vector [39]. This implies that the glide of a $1 / 2<111>$ screw dislocation in BCC W could be promoted as the $G_{111}$ decreases. Therefore, it is conceivable that the lattice softening induced by alloying elements may also result in a preferential softening for plastic deformation in a practical sense as well. Experimentally, Re has been recognized to strongly soften BCC W at room temperature, however, with alloying contents of over 5 at.\% $[3,4,40]$. In addition to Re, Ru, Os, or Ir have been reported to also have obvious softening effects on BCC W, but at much lower alloying levels (1 2 at.\%) [3]. It is interesting to note that at very a low alloying concentration (1.85 at.\%), the effect of Re on lattice softening (i.e., a decrease of $G_{111}$ ) is weaker compared to the effects of $\mathrm{Ru}$, Os and Ir, as shown by the present calculations (Table 1). However, the lattice softening induced by Re becomes much more significant as at concentrations greater than 5 at.\% [13]. As mentioned above, the alloying elements that have large disparities of the number of valence electrons compared to pure $\mathrm{W}$ will lead to significant decrease of $G_{111}$. Therefore, by taking the real solubility limits of alloy elements in BCC W into account, it is hypothesized that $\mathrm{Ti}, \mathrm{Rh}$, and Hf could be possibly more promising candidates to soften BCC $\mathrm{W}$ via alloying. 
Nevertheless, it needs to be pointed out that this hypothesis is purely based on the elastic properties of the host metal and alloying additions which may not be able to reflect the overall effects of alloying on plasticity. Further experiments or calculations incorporating a better plasticity model are needed to validate the current hypothesis.

\subsection{Elastic Properties at Finite Temperatures}

The elastic properties of pure $\mathrm{W}$ and $\mathrm{W}_{53} \mathrm{X}$ type alloys at finite temperatures are predicted via the quasi-static approach based on the Debye model presented in Section 2.2. The Debye temperature of pure $\mathrm{W}$ is calculated to be $363 \mathrm{~K}$ in the present work, which is in good agreement with the experimental value $(378 \mathrm{~K})$ and results of a previous calculation (366 K) [6]. Figure 8 plots the predicted isentropic and isothermal $c_{i j}$ 's and the bulk modulus of pure $\mathrm{W}$ from 0 to 3750 K. Available experimental data, measured in the isentropic condition, are also included for comparison $[22,28,41]$. It is shown that the predicted elastic constant values decrease with increasing temperature. This is caused by lattice expansion at high temperatures which leads to the weakening of atomic bonds. Computed values of the isothermal bulk modulus and $c_{44}$ agree more closely with the experimental data. A relatively large discrepancy is observed between experimental $c_{11}$ and the predicted values at high temperatures. This is possibly due to the strong anharmonicity in $\mathrm{W}$ at high temperatures or caused by the thermal stress fluctuations, which are not considered in the present work.

Table 2 summarizes the predicted isothermal and isentropic elastic constants of $\mathrm{W}_{53} \mathrm{X}$ at several selected temperatures, $300,900,1500,2100,2700$, and $3300 \mathrm{~K}$. It is worth mentioning that the value for the isothermal $c_{44}$ equals to the isentropic $c_{44}$ for a cubic crystal. Similarly to pure $\mathrm{W}$, the predicted $c_{i j}$ values of $\mathrm{W}_{53} \mathrm{X}$ decrease with increasing temperature. For the sake of 
applicability, the predicted $c_{i j}$ values of $\mathrm{W}_{53} \mathrm{X}$ from 300 to $3750 \mathrm{~K}$ are fitted to a polynomial function [11],

Equation 17

$$
c_{i j}(T)=a_{0}+a_{1} T+a_{2} T^{2}
$$

where $a_{0}, a_{1}$, and $a_{2}$ are fitting parameters. The fitting parameters obtained for each $\mathrm{W}_{53} \mathrm{X}$ are listed in Table 2. In addition, fitting errors are estimated by,

Equation 18

$$
\text { Error }=\sqrt{\frac{\sum\left[\left(c_{\text {fit }}-c_{\text {calc }}\right) / c_{\text {calc }}\right]^{2}}{n}}
$$

where $c_{\text {calc }}$ and $c_{f i t}$ are elastic stiffness constants predicted by the quasi-static approach and by Equation 17, respectively. These fitting errors are also included in Table 2. As seen in Table 2, the decreasing trend of elastic constants is indicated by $a_{l}$ roughly. The average values of $a_{l}$ for isothermal $c_{11}, c_{12}$ and $c_{44}$ are $-2.88 \times 10^{-2},-1.60 \times 10^{-2}$ and $-0.58 \times 10^{-2} \mathrm{GPa} / \mathrm{K}$, respectively. For isentropic $c_{11}$ and $c_{12}$, the average values of $a_{1}$ are $-1.65 \times 10^{-2}$ and $-0.56 \times 10^{-2}$, respectively.

\section{Conclusions}

In the present work, the effects of alloying elements and temperature on the elastic properties of BCC W, including isothermal and isentropic elastic stiffness constants, bulk modulus, shear modulus, Young's modulus and elastic anisotropy have been studied systematically via firstprinciples calculations. Nineteen transition metal alloying elements (X's) have been considered: Ti, V, Cr, Fe, Co, Ni, Y, Zr, Nb, Mo, Ru, Rh, Pd, Hf, Ta, Re, Os, Ir, and Pt. Based on the firstprinciples results at $0 \mathrm{~K}$, the alloying effects on the elasticity of $\mathrm{BCC} \mathrm{W}$ can be summarized as follows: (i) the bulk modulus of the dilute $\mathrm{W}-\mathrm{X}$ alloys decreases with increasing equilibrium volume, particularly for alloying elements in the same period; (ii) all alloying elements decrease 
the shear modulus of BCC W to some extent; (iii) the earlier or later transition elements (e.g., Y, $\mathrm{Zr}$ and $\mathrm{Pd}$ ) have stronger effects on the shear modulus than the other elements (e.g., $\mathrm{Cr}$ and $\mathrm{Mo}$ ) which have the same number of valence electrons as $\mathrm{W}$; and (iv) the largest decrease of elastic properties of $\mathrm{W}$ is due to the alloying element of $\mathrm{Y}$. In addition, effects of alloying elements on the elastic properties of $\mathrm{W}$, such as the bulk modulus and shear modulus, are traceable from the redistribution of electronic charge densities caused by alloying elements. At finite temperatures, the isothermal and isentropic elastic stiffness components of the W-X dilute alloys are predicted via a quasi-static approach based on the Debye model and show a decreasing trend of elasticity with increasing temperature due to lattice expansion at finite temperatures. Computed elastic properties are compared to available experimental data and are found to be in good agreement. 


\section{Acknowledgements:}

The financial support from the U.S. Army Research Laboratory under contract W911NF-082-0084 is greatly acknowledged. This work is also supported in part by a grant of computer time from the DoD High Performance Computing Modernization Program at U.S. Air Force Research Laboratory (ARFL) DoD Supercomputing Resource Center (DSRC). First-principles calculations also partially used the Extreme Science and Engineering Discovery Environment (XSEDE), which is supported by National Science Foundation Grant No. ACI-1053575, and were partially carried out on the LION clusters at the Pennsylvania State University supported by the Materials Simulation Center and the Institute for CyberScience. Lastly, we would like to thank Dr. Bi-Cheng Zhou and Dr. Xuan Liu in the Phases Research Laboratory at the Pennsylvania State University for stimulating discussions. 
Table 1. Calculated elastic properties of $\mathrm{W}_{53} \mathrm{X}$ alloys at $0 \mathrm{~K}$, including elastic stiffness constants $\left(c_{i j}\right.$ 's), Young's modulus $(E)$, bulk modulus $(B)$, and shear modulus $(G)$ from the Voigt approach, shear modulus along the [111] direction $\left(G_{111}\right)$, and the universal elastic anisotropy ratio $\left(A^{U}\right)$. The unit for each elastic property is GPa. The EOS predicted equilibrium volume $\left(V_{0}\left(\AA^{3} /\right.\right.$ atom $\left.)\right)$, the corresponding lattice parameter $\left(a_{0}(\AA)\right)$, and lattice regression coefficient $\left(k_{x}\right)$ are shown as well. For BCC W, available experimental data and previous calculations are also included for comparison.

\begin{tabular}{|c|c|c|c|c|c|c|c|c|c|c|c|}
\hline$X$ & $V_{0}$ & $a_{0}$ & $k_{x}$ & $c_{11}$ & $c_{12}$ & $c_{44}$ & $E$ & $B$ & $G$ & $G_{111}$ & $A^{U}$ \\
\hline $\mathrm{W}$ & $\begin{array}{c}16.226 \\
16.229[5]\end{array}$ & $\begin{array}{l}3.190 \\
3.190\end{array}$ & 0.000 & $\begin{array}{l}525.6 \\
517.8\end{array}$ & $\begin{array}{l}192.8 \\
201.7\end{array}$ & $\begin{array}{l}143.3 \\
139.4\end{array}$ & 392.0 & 303.7 & 152.5 & 157.9 & 0.027 \\
\hline Expt.[28] & 15.856 & 3.165 & 0.000 & 533.9 & 205.1 & 163.3 & 418.6 & 314.7 & 163.7 & 164.0 & 0.000 \\
\hline $\mathrm{Ti}$ & 16.206 & 3.188 & -0.070 & 507.3 & 195.7 & 128.7 & 362.4 & 299.5 & 139.6 & 145.6 & 0.044 \\
\hline $\mathrm{V}$ & 16.183 & 3.187 & -0.154 & 515.6 & 193.7 & 136.1 & 377.1 & 301.0 & 146.0 & 151.7 & 0.034 \\
\hline $\mathrm{Cr}$ & 16.160 & 3.185 & -0.235 & 521.8 & 191.9 & 142.4 & 389.2 & 301.9 & 151.4 & 156.7 & 0.026 \\
\hline $\mathrm{Fe}$ & 16.149 & 3.185 & -0.271 & 503.2 & 203.4 & 135.7 & 367.1 & 303.3 & 141.4 & 144.8 & 0.012 \\
\hline Co & 16.150 & 3.185 & -0.268 & 499.0 & 202.3 & 135.3 & 364.9 & 301.2 & 140.5 & 143.7 & 0.010 \\
\hline $\mathrm{Ni}$ & 16.160 & 3.185 & -0.233 & 498.7 & 205.1 & 136.4 & 365.3 & 302.9 & 140.6 & 143.2 & 0.006 \\
\hline Y & 16.346 & 3.198 & 0.423 & 495.8 & 191.2 & 128.9 & 358.3 & 292.7 & 138.2 & 143.6 & 0.034 \\
\hline $\mathrm{Zr}$ & 16.300 & 3.195 & 0.263 & 493.4 & 197.3 & 126.1 & 351.3 & 296.0 & 134.9 & 139.9 & 0.031 \\
\hline $\mathrm{Nb}$ & 16.255 & 3.192 & 0.103 & 517.2 & 192.9 & 138.7 & 381.6 & 301.0 & 148.1 & 153.5 & 0.029 \\
\hline Mo & 16.220 & 3.189 & -0.020 & 523.4 & 192.4 & 141.9 & 389.1 & 302.7 & 151.3 & 156.8 & 0.029 \\
\hline
\end{tabular}




\begin{tabular}{llllllllllll}
$\mathrm{Ru}$ & 16.190 & 3.187 & -0.126 & 505.2 & 201.5 & 136.9 & 370.4 & 302.7 & 142.9 & 146.5 & 0.013 \\
$\mathrm{Rh}$ & 16.209 & 3.189 & -0.062 & 497.2 & 203.6 & 135.0 & 363.1 & 301.5 & 139.7 & 142.6 & 0.008 \\
$\mathrm{Pd}$ & 16.222 & 3.189 & -0.015 & 491.5 & 205.4 & 135.0 & 359.7 & 300.8 & 138.3 & 140.3 & 0.004 \\
$\mathrm{Hf}$ & 16.285 & 3.194 & 0.208 & 505.8 & 194.5 & 130.4 & 364.2 & 298.3 & 140.5 & 146.2 & 0.038 \\
$\mathrm{Ta}$ & 16.254 & 3.192 & 0.098 & 517.2 & 193.9 & 138.6 & 381.2 & 301.7 & 147.8 & 153.1 & 0.028 \\
$\mathrm{Re}$ & 16.204 & 3.188 & -0.079 & 523.4 & 195.2 & 143.2 & 390.0 & 304.6 & 151.6 & 156.5 & 0.022 \\
$\mathrm{Os}$ & 16.193 & 3.188 & -0.118 & 512.8 & 201.2 & 142.7 & 382.1 & 305.1 & 147.9 & 151.2 & 0.009 \\
$\mathrm{Ir}$ & 16.201 & 3.188 & -0.088 & 501.8 & 204.9 & 139.1 & 370.5 & 303.9 & 142.8 & 145.2 & 0.005 \\
$\mathrm{Pt}$ & 16.224 & 3.190 & -0.009 & 492.6 & 206.1 & 138.3 & 364.4 & 301.6 & 140.3 & 141.6 & 0.001 \\
\hline
\end{tabular}


Table 2. Predicted isothermal elastic stiffness constant $\left(c_{i j}{ }^{T}\right)$ and isentropic elastic stiffness constant $\left(c_{i j}{ }^{S}\right)$ of $\mathrm{W}_{53} \mathrm{X}$ at $300,900,1500$, 2100, 2700, and $3300 \mathrm{~K}$. The unit for the elastic stiffness constants is GPa. The polynomial fitting parameters and fitting errors are also included; their values are based on the predicted elastic data from 300 to $3300 \mathrm{~K}$.

\begin{tabular}{|c|c|c|c|c|c|c|c|c|c|c|c|}
\hline $\mathrm{X}$ & $c_{i j}$ & $T_{300}$ & $T_{900}$ & $T_{1500}$ & $T_{2100}$ & $T_{2700}$ & $T_{3300}$ & $a_{0}$ & $a_{1} \times 10^{2}$ & $a_{2} \times 10^{6}$ & error $\times 10^{4}$ \\
\hline \multirow[t]{5}{*}{$\bar{W}$} & $c_{11}^{T}$ & 516.9 & 500.0 & 482.2 & 463.6 & 444.1 & 423.2 & 524.7 & -2.62 & -1.40 & 4.1 \\
\hline & $c_{12}^{T}$ & 186.4 & 175.6 & 164.9 & 154.5 & 144.3 & 134.5 & 192.2 & -1.88 & 0.40 & 4.6 \\
\hline & $c_{44}{ }^{T}$ & 141.1 & 136.7 & 131.9 & 126.7 & 121.0 & 114.7 & 142.9 & -0.62 & -0.70 & 7.8 \\
\hline & $c_{11}^{S}$ & 519.5 & 508.0 & 495.0 & 480.8 & 465.4 & 448.7 & 525.1 & -1.75 & -1.72 & 1.9 \\
\hline & $c_{12}{ }^{S}$ & 189.0 & 183.6 & 177.6 & 171.6 & 165.7 & 160.0 & 192.5 & -1.01 & 0.08 & 11.2 \\
\hline \multirow[t]{5}{*}{$\mathrm{Ti}$} & $c_{11}^{T}$ & 497.5 & 479.6 & 461.0 & 442.0 & 422.4 & 401.7 & 506.0 & -2.85 & -0.95 & 3.2 \\
\hline & $c_{12}^{T}$ & 190.0 & 179.1 & 168.3 & 157.3 & 146.2 & 134.9 & 195.1 & -1.76 & -0.20 & 5.3 \\
\hline & $c_{44}{ }^{T}$ & 126.2 & 121.7 & 117.0 & 112.2 & 107.2 & 101.9 & 128.3 & -0.71 & -0.26 & 6.6 \\
\hline & $c_{11}^{S}$ & 500.4 & 488.5 & 475.2 & 461.1 & 446.2 & 430.2 & 506.3 & -1.87 & -1.33 & 2.2 \\
\hline & $c_{12}{ }^{S}$ & 192.7 & 188.1 & 182.5 & 176.4 & 170.1 & 163.4 & 195.5 & -0.78 & -0.58 & 10.0 \\
\hline \multirow[t]{5}{*}{$\mathrm{V}$} & $c_{11}^{T}$ & 505.8 & 487.6 & 468.9 & 449.8 & 430.1 & 409.7 & 514.6 & -2.92 & -0.76 & 3.6 \\
\hline & $c_{12}^{T}$ & 188.3 & 178.5 & 168.4 & 158.3 & 147.9 & 137.2 & 193.2 & -1.61 & -0.28 & 8.8 \\
\hline & $c_{44}^{T}$ & 133.5 & 128.5 & 123.4 & 118.1 & 112.7 & 107.0 & 135.9 & -0.80 & -0.24 & 7.4 \\
\hline & $c_{11}^{S}$ & 508.5 & 495.9 & 482.2 & 467.7 & 452.5 & 436.3 & 514.6 & -1.97 & -1.22 & 1.7 \\
\hline & $c_{12}{ }^{S}$ & 191.0 & 186.8 & 181.8 & 176.2 & 170.3 & 163.8 & 193.2 & -0.65 & -0.74 & 5.4 \\
\hline \multirow[t]{5}{*}{$\mathrm{Cr}$} & $c_{11}^{T}$ & 512.8 & 495.6 & 477.4 & 458.4 & 438.3 & 416.8 & 520.7 & -2.64 & -1.55 & 3.0 \\
\hline & $c_{12}^{T}$ & 186.0 & 175.4 & 164.7 & 154.0 & 143.5 & 132.9 & 191.4 & -1.79 & -0.04 & 6.2 \\
\hline & $c_{44^{T}}$ & 140.6 & 136.3 & 131.5 & 126.1 & 120.2 & 113.4 & 142.3 & -0.58 & -0.91 & 9.5 \\
\hline & $c_{11}^{S}$ & 515.6 & 504.3 & 491.4 & 477.1 & 461.5 & 444.8 & 521.3 & -1.73 & -1.77 & 3.5 \\
\hline & $c_{12}{ }^{S}$ & 188.8 & 184.1 & 178.7 & 172.8 & 166.6 & 160.9 & 192.1 & -0.88 & -0.18 & 18.8 \\
\hline \multirow[t]{5}{*}{$\mathrm{Fe}$} & $c_{11}^{T}$ & 496.8 & 480.1 & 462.2 & 443.6 & 423.8 & 402.7 & 504.7 & -2.59 & -1.51 & 2.5 \\
\hline & $c_{12}{ }^{T}$ & 193.1 & 182.2 & 171.2 & 160.3 & 149.4 & 138.7 & 198.8 & -1.86 & -0.10 & 7.7 \\
\hline & $c_{44^{T}}$ & 135.2 & 131.1 & 126.5 & 121.4 & 115.7 & 109.2 & 136.8 & -0.55 & -0.87 & 6.1 \\
\hline & $c_{11}^{S}$ & 499.6 & 488.6 & 476.0 & 462.2 & 447.2 & 430.3 & 504.5 & -1.58 & -2.05 & 4.1 \\
\hline & $c_{12}{ }^{S}$ & 195.9 & 190.8 & 185.0 & 178.9 & 172.8 & 166.3 & 198.6 & -0.84 & -0.43 & 5.9 \\
\hline
\end{tabular}




\begin{tabular}{|c|c|c|c|c|c|c|c|c|c|c|c|}
\hline \multirow[t]{5}{*}{ Co } & $c_{11}^{T}$ & 488.9 & 470.4 & 451.5 & 432.6 & 413.7 & 394.6 & 498.4 & -3.10 & -0.13 & 2.4 \\
\hline & $c_{12}^{T}$ & 195.8 & 184.6 & 173.2 & 161.9 & 150.7 & 139.5 & 201.7 & -1.91 & 0.08 & 4.1 \\
\hline & $c_{44}^{T}$ & 132.9 & 128.0 & 123.0 & 117.8 & 112.4 & 106.6 & 135.2 & -0.76 & -0.31 & 7.8 \\
\hline & $c_{11} S$ & 491.7 & 479.1 & 465.6 & 451.5 & 437.3 & 422.8 & 498.6 & -2.13 & -0.51 & 4.2 \\
\hline & $c_{12}{ }^{S}$ & 198.6 & 193.3 & 187.3 & 180.8 & 174.3 & 167.8 & 201.9 & -0.94 & -0.30 & 11.3 \\
\hline \multirow[t]{5}{*}{$\mathrm{Ni}$} & $c_{11}^{T}$ & 489.0 & 470.8 & 452.0 & 432.7 & 412.9 & 392.4 & 497.9 & -2.94 & -0.78 & 0.8 \\
\hline & $c_{12}{ }^{T}$ & 199.0 & 187.7 & 176.2 & 164.7 & 153.1 & 141.5 & 204.8 & -1.90 & -0.06 & 3.4 \\
\hline & $c_{44}{ }^{T}$ & 134.0 & 129.2 & 124.1 & 118.8 & 113.1 & 107.0 & 136.1 & -0.73 & -0.47 & 8.5 \\
\hline & $c_{11} s$ & 491.8 & 479.6 & 466.0 & 451.6 & 436.2 & 419.8 & 497.9 & -1.92 & -1.34 & 2.7 \\
\hline & $c_{12} S$ & 201.9 & 196.5 & 190.3 & 183.6 & 176.4 & 169.0 & 204.9 & -0.88 & -0.62 & 8.6 \\
\hline \multirow[t]{5}{*}{$\mathrm{Y}$} & $c_{11}^{T}$ & 485.5 & 465.9 & 445.9 & 425.6 & 405.0 & 383.9 & 495.1 & -3.20 & -0.52 & 1.2 \\
\hline & $c_{12}^{T}$ & 185.8 & 176.1 & 166.1 & 156.0 & 145.4 & 134.5 & 190.5 & -1.55 & -0.43 & 8.0 \\
\hline & $c_{44}{ }^{T}$ & 125.8 & 120.0 & 114.2 & 108.3 & 102.2 & 96.1 & 128.6 & -0.94 & -0.13 & 4.3 \\
\hline & $c_{11}^{44} S$ & 488.3 & 474.5 & 459.5 & 444.0 & 428.0 & 411.0 & 495.2 & -2.22 & -1.00 & 2.6 \\
\hline & $c_{12} s$ & 188.6 & 184.7 & 179.8 & 174.3 & 168.4 & 161.6 & 190.6 & -0.58 & -0.92 & 7.2 \\
\hline \multirow[t]{5}{*}{$\mathrm{Zr}$} & $c_{11}^{T}$ & 484.9 & 468.6 & 451.5 & 433.7 & 414.7 & 394.0 & 491.9 & -2.43 & -1.64 & 6.0 \\
\hline & $c_{12}^{T}$ & 191.7 & 181.1 & 170.5 & 160.2 & 150.0 & 139.8 & 197.0 & -1.78 & 0.13 & 5.3 \\
\hline & $c_{44}{ }^{T}$ & 124.4 & 120.5 & 116.5 & 112.3 & 107.9 & 103.1 & 126.0 & -0.58 & -0.33 & 9.3 \\
\hline & $c_{11}^{S}$ & 487.6 & 476.9 & 464.6 & 451.1 & 436.4 & 420.5 & 493.0 & -1.65 & -1.66 & 2.9 \\
\hline & $c_{12}{ }^{S}$ & 194.4 & 189.3 & 183.6 & 177.6 & 171.7 & 166.3 & 198.1 & -1.00 & -0.12 & 17.4 \\
\hline \multirow[t]{5}{*}{$\mathrm{Nb}$} & $c_{11}^{T}$ & 508.5 & 490.7 & 472.2 & 453.2 & 433.4 & 412.7 & 517.0 & -2.83 & -1.01 & 2.2 \\
\hline & $c_{12}^{T}$ & 187.1 & 177.1 & 167.0 & 156.9 & 146.7 & 136.5 & 192.2 & -1.68 & -0.03 & 4.8 \\
\hline & $c_{44}{ }^{T}$ & 136.3 & 130.9 & 125.3 & 119.7 & 113.8 & 107.6 & 138.9 & -0.86 & -0.26 & 5.3 \\
\hline & $c_{11} S$ & 511.1 & 498.9 & 485.3 & 470.7 & 455.3 & 438.3 & 516.8 & -1.86 & -1.59 & 3.5 \\
\hline & $c_{12}{ }^{S}$ & 189.8 & 185.3 & 180.0 & 174.4 & 168.6 & 162.1 & 192.1 & -0.71 & -0.61 & 5.4 \\
\hline \multirow[t]{5}{*}{ Mo } & $c_{11}^{T}$ & 515.0 & 498.8 & 481.4 & 463.0 & 443.3 & 421.9 & 522.2 & -2.43 & -1.85 & 3.2 \\
\hline & $c_{12}{ }^{T}$ & 186.4 & 175.7 & 165.0 & 154.4 & 143.9 & 133.6 & 192.0 & -1.83 & 0.18 & 3.3 \\
\hline & $c_{44}{ }^{T}$ & 140.0 & 135.7 & 131.1 & 126.0 & 120.4 & 114.0 & 141.7 & -0.59 & -0.76 & 7.8 \\
\hline & $c_{11} S$ & 517.7 & 507.1 & 494.8 & 481.0 & 465.8 & 448.4 & 522.1 & -1.45 & -2.38 & 3.1 \\
\hline & $c_{12}{ }^{S}$ & 189.1 & 184.0 & 178.3 & 172.4 & 166.4 & 160.0 & 191.9 & -0.85 & -0.35 & 5.7 \\
\hline \multirow[t]{3}{*}{$\mathrm{Ru}$} & $c_{11}^{T}$ & 495.2 & 476.9 & 458.0 & 438.7 & 419.0 & 398.6 & 504.2 & -2.98 & -0.69 & 4.1 \\
\hline & $c_{12}^{T}$ & 195.7 & 185.2 & 174.4 & 163.5 & 152.4 & 141.2 & 201.0 & -1.75 & -0.21 & 10.3 \\
\hline & $c_{44}^{T}$ & 134.1 & 128.8 & 123.3 & 117.8 & 112.1 & 106.2 & 136.7 & -0.86 & -0.19 & 3.1 \\
\hline
\end{tabular}




\begin{tabular}{|c|c|c|c|c|c|c|c|c|c|c|c|}
\hline & $c_{11}^{S}$ & 498.0 & 485.6 & 472.0 & 457.7 & 442.7 & 426.9 & 504.5 & -2.01 & -1.04 & 3.2 \\
\hline & $c_{12}{ }^{S}$ & 198.5 & 193.9 & 188.4 & 182.4 & 176.1 & 169.6 & 201.3 & -0.78 & -0.56 & 9.4 \\
\hline \multirow[t]{5}{*}{$\mathrm{Rh}$} & $c_{11}^{T}$ & 488.4 & 471.4 & 453.6 & 435.3 & 416.1 & 395.8 & 496.4 & -2.67 & -1.15 & 2.5 \\
\hline & $c_{12}^{T}{ }_{T}^{T}$ & 197.6 & 186.7 & 175.6 & 164.5 & 153.5 & 142.4 & 203.2 & -1.84 & 0.03 & 14.3 \\
\hline & $c_{44}{ }^{T}$ & 133.0 & 129.1 & 124.8 & 119.9 & 114.4 & 108.1 & 134.6 & -0.52 & -0.85 & 6.3 \\
\hline & $c_{11}^{S}$ & 491.1 & 480.0 & 467.4 & 453.8 & 439.2 & 423.1 & 496.4 & -1.67 & -1.66 & 3.1 \\
\hline & $c_{12}{ }^{s}$ & 200.4 & 195.2 & 189.4 & 183.1 & 176.6 & 169.8 & 203.2 & -0.85 & -0.51 & 7.1 \\
\hline \multirow[t]{5}{*}{$\mathrm{Pd}$} & $c_{11}^{T}$ & 481.8 & 463.4 & 444.4 & 425.2 & 405.6 & 385.5 & 491.0 & -3.02 & -0.54 & 1.4 \\
\hline & $c_{12}{ }^{T}$ & 199.4 & 188.4 & 177.2 & 165.8 & 154.4 & 142.9 & 205.0 & -1.84 & -0.14 & 6.1 \\
\hline & $c_{44}^{T}$ & 132.6 & 127.8 & 122.7 & 117.4 & 111.9 & 106.1 & 134.9 & -0.77 & -0.32 & 7.5 \\
\hline & $c_{11}{ }^{S}$ & 484.6 & 472.3 & 458.7 & 444.4 & 429.5 & 414.2 & 491.3 & -2.05 & -0.88 & 4.6 \\
\hline & $c_{12}{ }^{S}$ & 202.3 & 197.3 & 191.4 & 185.1 & 178.4 & 171.6 & 205.4 & -0.86 & -0.48 & 12.8 \\
\hline \multirow[t]{5}{*}{$\mathrm{Hf}$} & $c_{11}^{T}$ & 496.1 & 478.3 & 460.0 & 441.3 & 422.1 & 402.0 & 504.6 & -2.84 & -0.83 & 3.1 \\
\hline & $c_{12}{ }^{T}$ & 188.9 & 178.5 & 168.0 & 157.5 & 146.9 & 136.1 & 194.0 & -1.71 & -0.12 & 5.8 \\
\hline & $c_{44}{ }^{T}$ & 127.4 & 122.3 & 117.1 & 111.8 & 106.6 & 101.2 & 130.0 & -0.85 & -0.07 & 3.2 \\
\hline & $c_{11}^{S}$ & 498.8 & 486.7 & 473.3 & 459.2 & 444.4 & 428.7 & 505.1 & -1.96 & -1.08 & 3.0 \\
\hline & $c_{12}{ }^{S}$ & 191.6 & 186.9 & 181.3 & 175.4 & 169.2 & 162.8 & 194.6 & -0.83 & -0.38 & 13.6 \\
\hline \multirow[t]{5}{*}{$\mathrm{Ta}$} & $c_{11}^{T}$ & 508.2 & 491.0 & 473.1 & 454.5 & 434.9 & 414.2 & 516.1 & -2.66 & -1.30 & 2.6 \\
\hline & $c_{12}{ }^{T}$ & 188.2 & 177.7 & 167.1 & 156.7 & 146.2 & 135.8 & 193.5 & -1.76 & -0.05 & 7.1 \\
\hline & $c_{44}{ }^{2}$ & 136.2 & 131.6 & 126.7 & 121.4 & 115.6 & 109.1 & 138.1 & -0.65 & -0.68 & 8.2 \\
\hline & $c_{11}^{4+4} S$ & 510.9 & 499.4 & 486.4 & 472.4 & 457.2 & 440.9 & 516.6 & -1.78 & -1.56 & 2.0 \\
\hline & $c_{12} s$ & 190.8 & 186.0 & 180.4 & 174.5 & 168.5 & 162.5 & 194.0 & -0.88 & -0.22 & 12.6 \\
\hline \multirow[t]{5}{*}{$\operatorname{Re}$} & $c_{11}^{T}$ & 513.7 & 496.0 & 477.4 & 458.2 & 438.0 & 416.8 & 522.2 & -2.79 & -1.23 & 2.1 \\
\hline & $c_{12}^{T}$ & 189.2 & 178.2 & 167.0 & 155.9 & 145.0 & 134.1 & 195.0 & -1.88 & -0.09 & 6.8 \\
\hline & $c_{44}{ }^{12}$ & 140.3 & 134.9 & 129.2 & 123.5 & 117.6 & 111.6 & 143.0 & -0.89 & -0.18 & 4.0 \\
\hline & $c_{11} S$ & 516.6 & 504.8 & 491.6 & 477.3 & 461.8 & 445.5 & 522.5 & -1.84 & -1.50 & 2.4 \\
\hline & $c_{12} s$ & 192.0 & 186.9 & 181.2 & 175.1 & 168.8 & 162.7 & 195.3 & -0.93 & -0.18 & 12.8 \\
\hline \multirow[t]{5}{*}{ Os } & $c_{11}^{T}$ & 501.5 & 477.8 & 454.7 & 432.6 & 411.7 & 392.2 & 514.6 & -4.24 & 1.61 & 4.7 \\
\hline & $c_{12}{ }^{T}$ & 195.8 & 185.1 & 174.2 & 163.2 & 152.2 & 141.1 & 201.3 & -1.79 & -0.10 & 3.5 \\
\hline & $c_{44}{ }^{T}$ & 138.7 & 132.0 & 125.3 & 118.8 & 112.6 & 106.6 & 142.4 & -1.19 & 0.31 & 6.9 \\
\hline & $c_{11}{ }^{S}$ & 504.3 & 486.7 & 468.7 & 451.3 & 434.9 & 419.7 & 514.8 & -3.26 & 1.15 & 7.9 \\
\hline & $c_{12}{ }^{S}$ & 198.7 & 193.9 & 188.2 & 182.0 & 175.4 & 168.6 & 201.6 & -0.81 & -0.57 & 9.9 \\
\hline $\mathrm{Ir}$ & $c_{11}^{T}$ & 491.6 & 473.2 & 454.1 & 434.6 & 414.5 & 393.7 & 500.5 & -2.96 & -0.85 & 2.1 \\
\hline
\end{tabular}




\begin{tabular}{|c|c|c|c|c|c|c|c|c|c|c|c|}
\hline \multirow{9}{*}{$\mathrm{Pt}$} & $c_{12}^{T}$ & 199.2 & 188.7 & 178.0 & 167.2 & 156.4 & 145.5 & 204.5 & -1.75 & -0.13 & 5.6 \\
\hline & $c_{44}^{T}$ & 135.8 & 130.3 & 124.5 & 118.5 & 112.2 & 105.7 & 138.5 & -0.88 & -0.34 & 3.5 \\
\hline & $c_{11}^{S}$ & 494.3 & 481.7 & 467.9 & 453.1 & 437.5 & 421.2 & 500.8 & -2.03 & -1.15 & 3.2 \\
\hline & $c_{12}{ }^{S}$ & 201.9 & 197.2 & 191.7 & 185.7 & 179.4 & 173.0 & 204.8 & -0.82 & -0.44 & 9.7 \\
\hline & $c_{11}^{T}$ & 482.9 & 464.8 & 446.2 & 427.1 & 407.6 & 387.4 & 491.7 & -2.92 & -0.72 & 1.7 \\
\hline & $c_{12}^{T}$ & 200.3 & 189.7 & 178.9 & 167.9 & 156.7 & 145.3 & 205.6 & -1.75 & -0.25 & 8.1 \\
\hline & $c_{44}^{T}$ & 135.5 & 130.2 & 124.7 & 119.1 & 113.4 & 107.5 & 138.1 & -0.86 & -0.20 & 3.1 \\
\hline & $c_{11}^{S}$ & 485.6 & 473.5 & 460.2 & 446.1 & 431.3 & 415.9 & 492.0 & -1.97 & -1.01 & 3.8 \\
\hline & $c_{12}{ }^{S}$ & 203.1 & 198.4 & 192.9 & 186.8 & 180.4 & 173.8 & 205.9 & -0.80 & -0.54 & 10.0 \\
\hline
\end{tabular}


Figure 1. Calculated equilibrium volumes of the $\mathrm{W}_{53} \mathrm{X}$ alloys (listed in Table 1) compared to the predictions from Vegard's law of pure elements with a BCC structure [5]. The dashed line indicates where the two methods produce the same result.

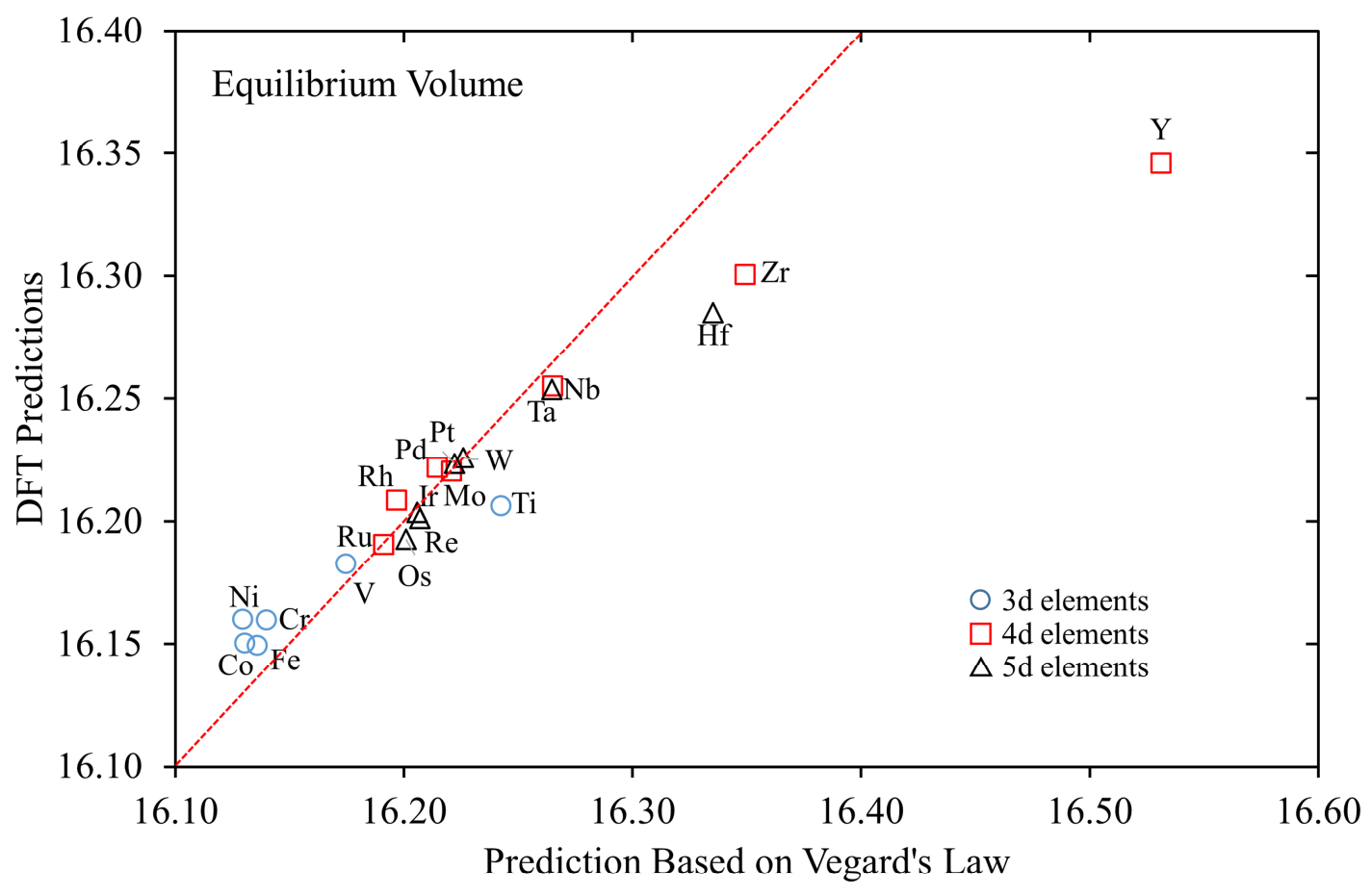


Figure 2. Calculated lattice regression coefficients $\left(k_{x}\right)$ of the $\mathrm{W}_{53} \mathrm{X}$ alloys (see Equation 15) vs. lattice parameters of the alloying elements $(\mathrm{Xs})$ in the BCC structure [5]. The $k_{x}$ values fitted from experimental data are labeled as black dots [31,32].

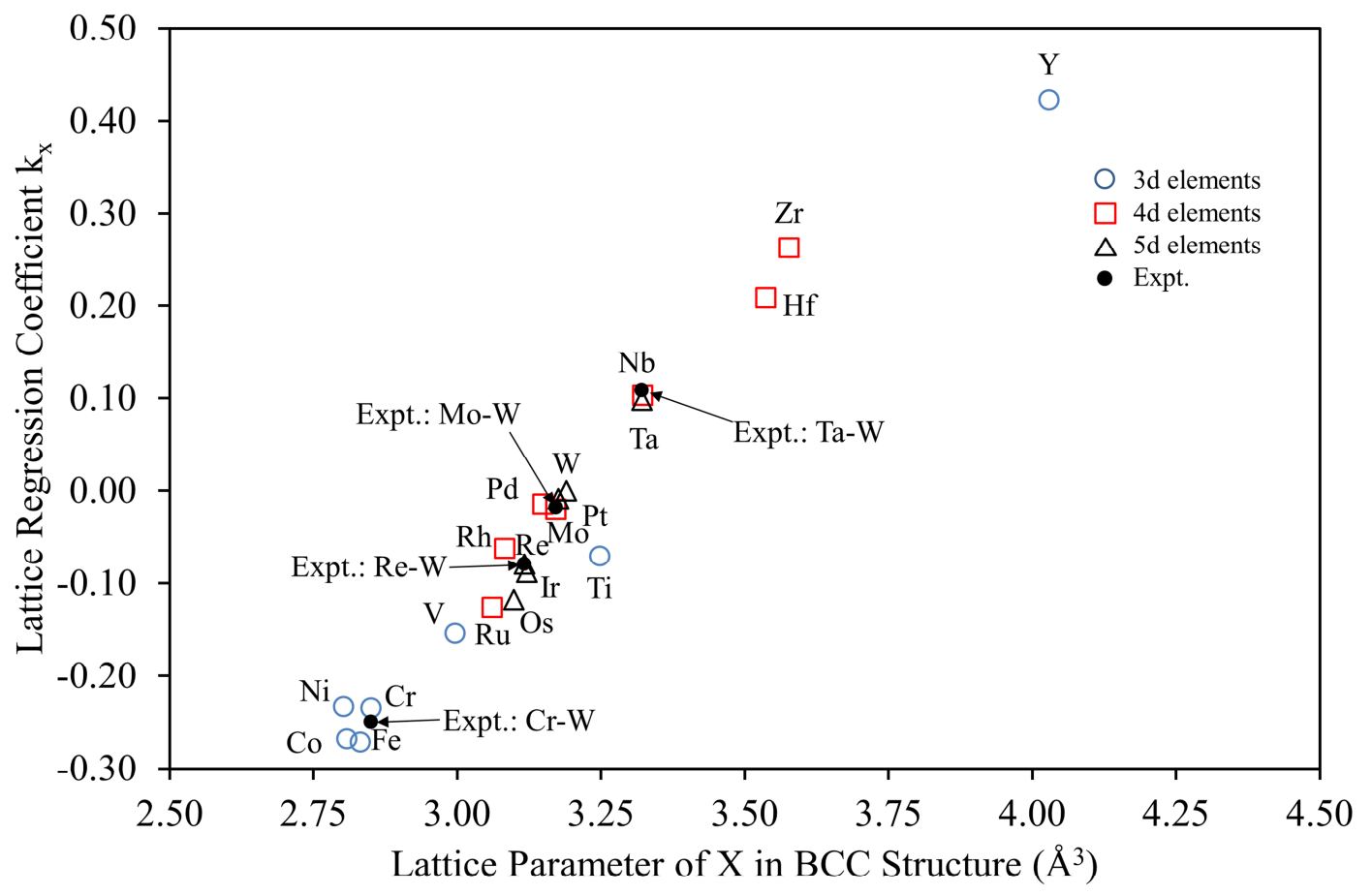


Figure 3. Bulk modulus of the $\mathrm{W}_{53} \mathrm{X}$ alloys (listed in Table 1) compared to the predictions from a linear combination of bulk moduli of the pure elements in the BCC structure in terms of their mole fractions [5]. The dashed line indicates where the two methods agree.

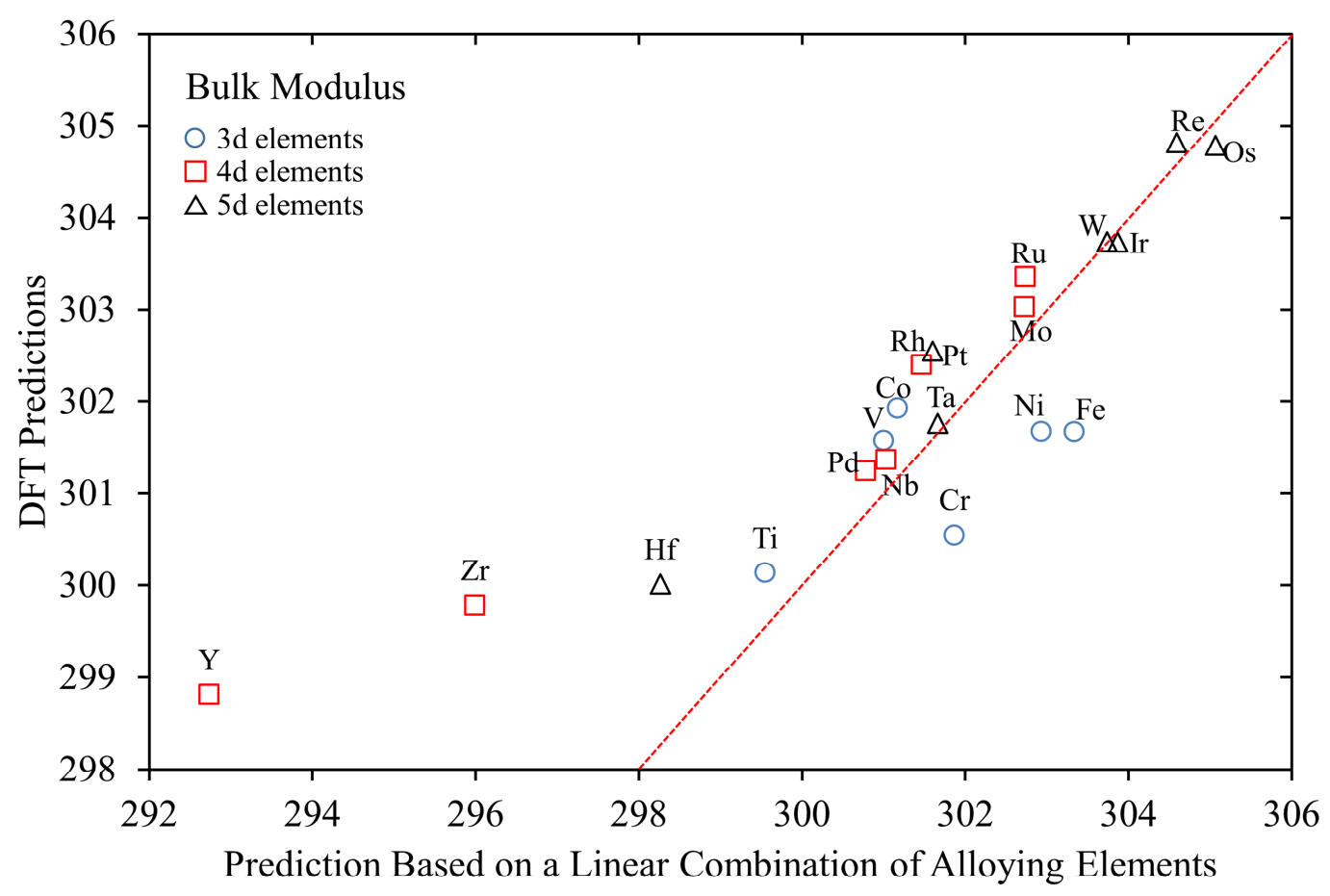


Figure 4. Calculated bulk and shear moduli of the $\mathrm{W}_{53} \mathrm{X}$ alloys based on the Voigt approach as a function of the equilibrium volume; (a) bulk modulus and (b) shear modulus.

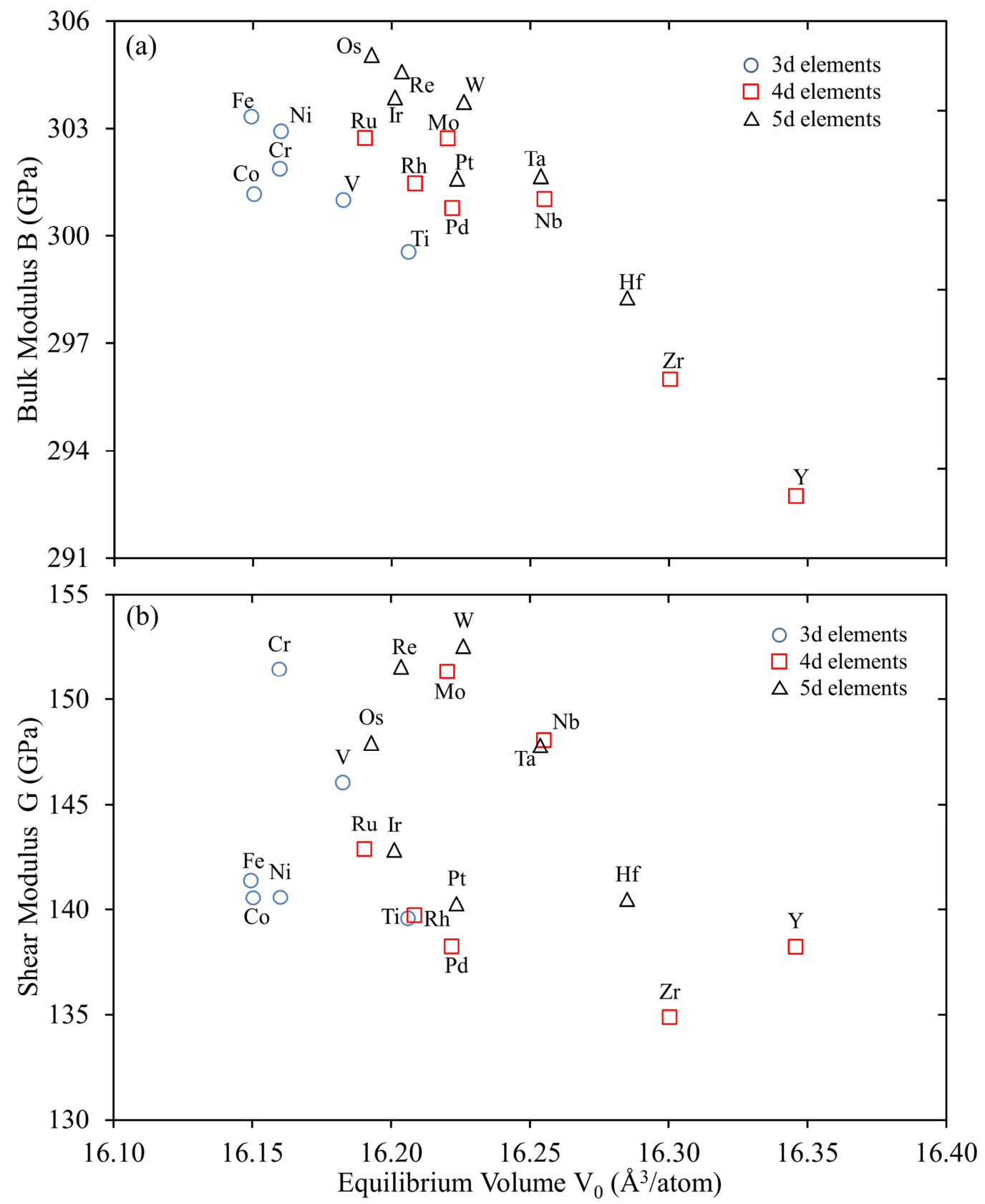


Figure 5. Calculated shear moduli of the $\mathrm{W}_{53} \mathrm{X}$ alloys with respect to the number of the valence electrons of the alloying elements.

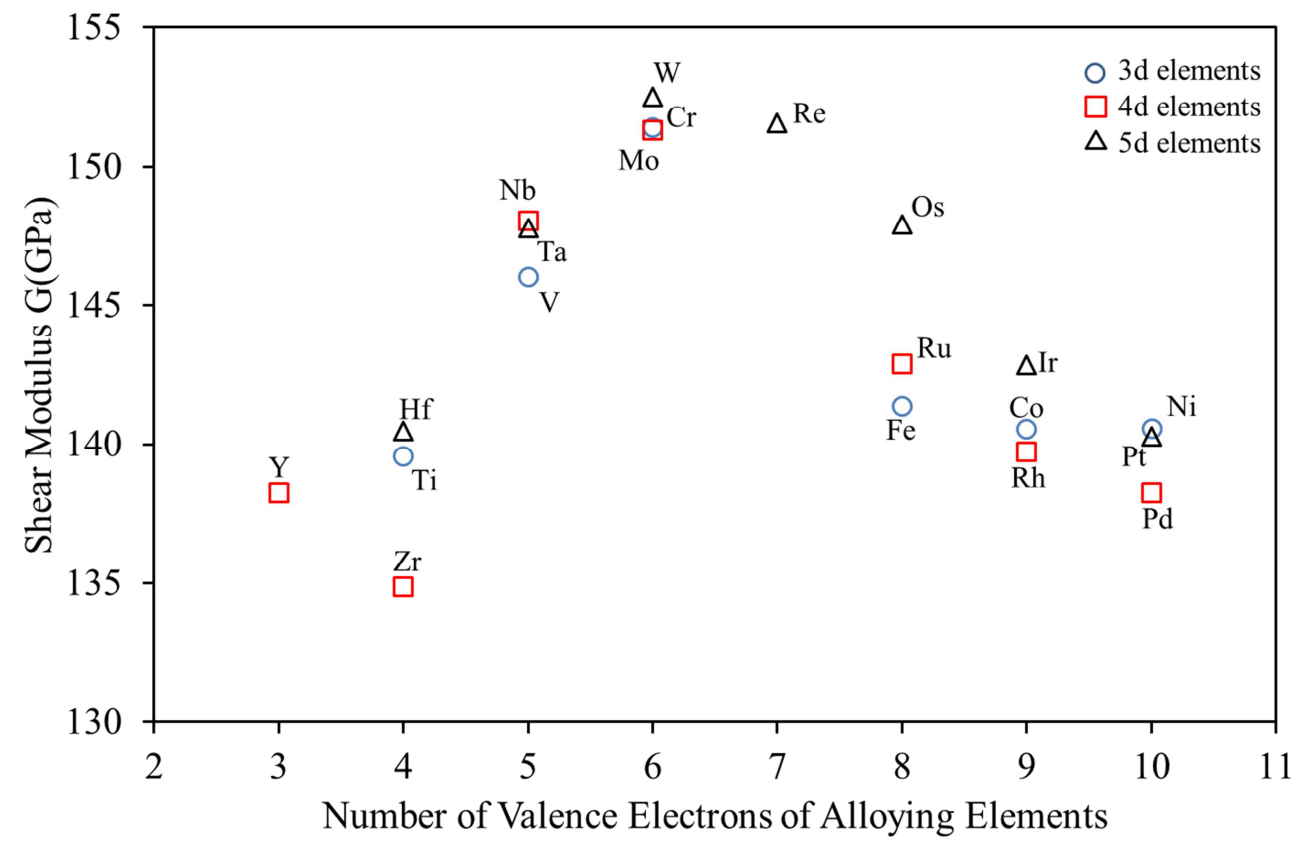


Figure 6. Correlation between $n$ and $\sqrt{B / V_{m}}$ for the $\mathrm{W}_{53} \mathrm{X}$ alloys.

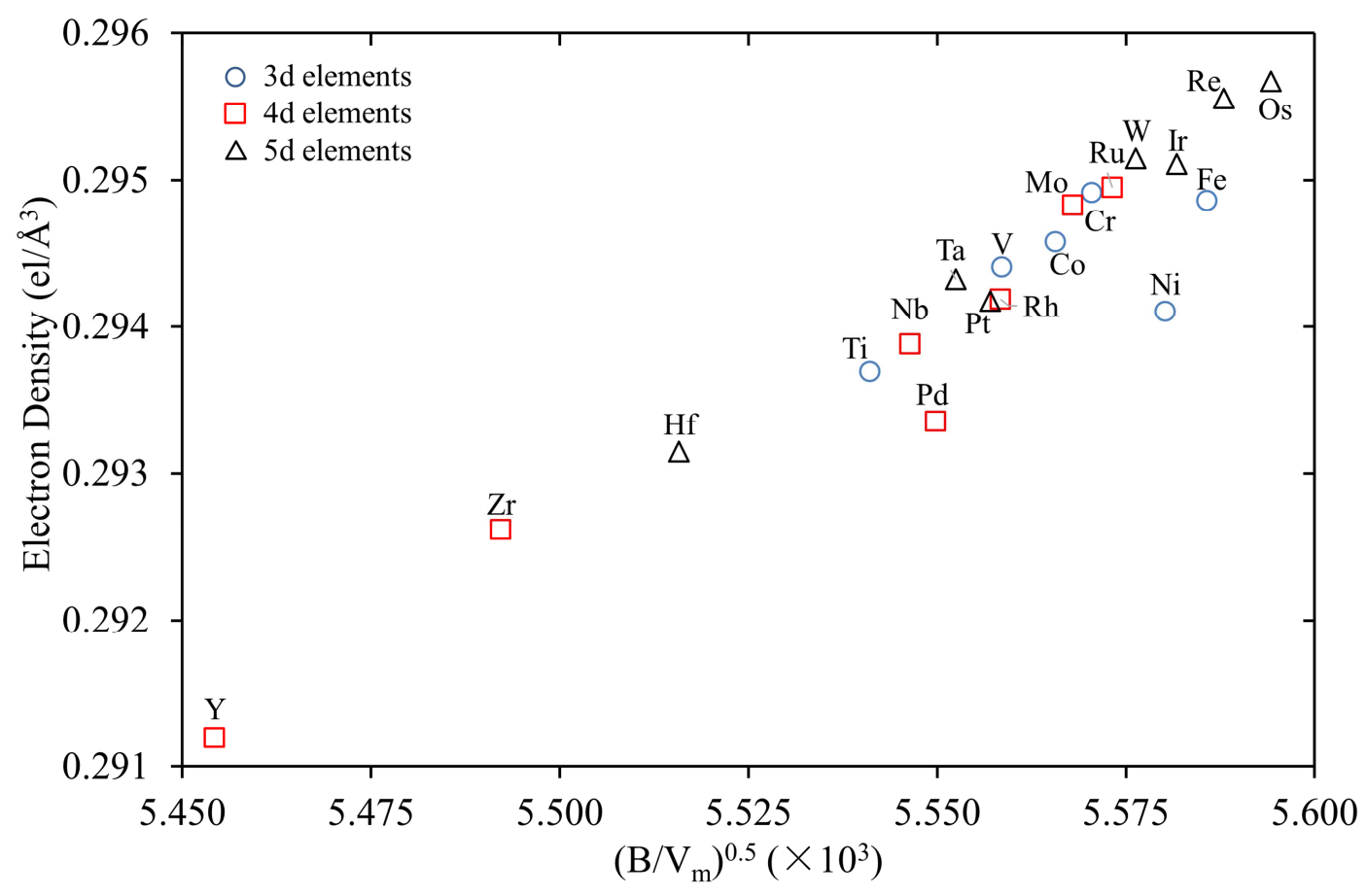


Figure 7. Differential charge densities of pure $\mathrm{BCC} W$ and the $\mathrm{W}_{53} \mathrm{X}$ alloys in the (11) plane; (a) pure BCC W, (b) $\mathrm{W}_{53} \mathrm{Mo}$, (c) $\mathrm{W}_{53} \mathrm{Ti}$, and (d) $\mathrm{W}_{53} \mathrm{Pd}$. The shortest distance between two $\mathrm{W}$ atoms or the solute and $\mathrm{W}$ atom is shown by dashed arrows. Note, the lattice indices in the figures are with respect to the 54-atom supercell. The unit of charge density is e/ $\AA^{3}$. Contour lines are spaced at $0.002 \mathrm{e} / \AA^{3}$.

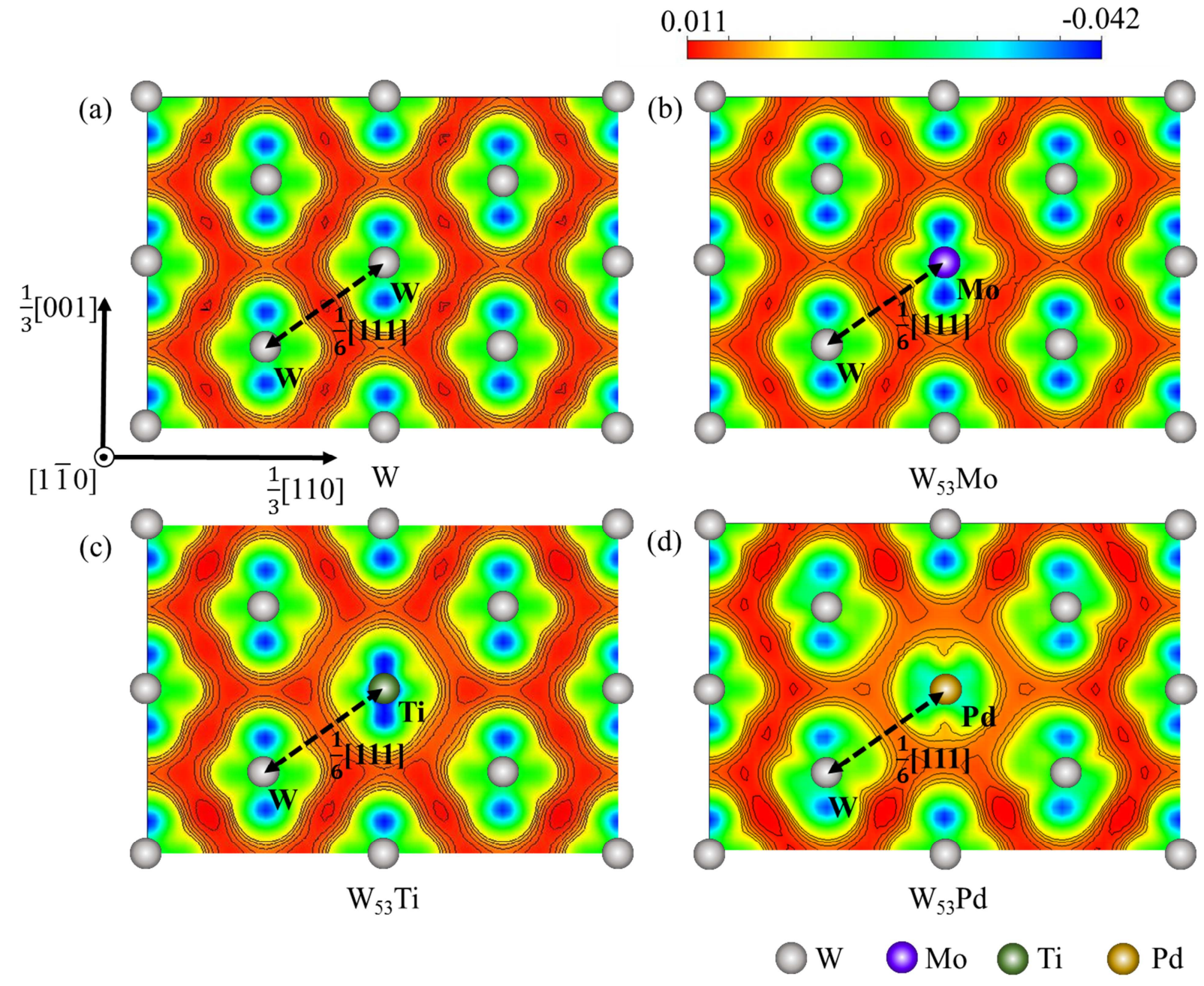


Figure 8. Predicted isentropic and isothermal $\mathrm{c}_{\mathrm{ij}}$ values of pure $\mathrm{W}$ from 0 to $3750 \mathrm{~K}$. Available experimental data are also included for comparison $(\square[28], \circ[22], \Delta[41])$

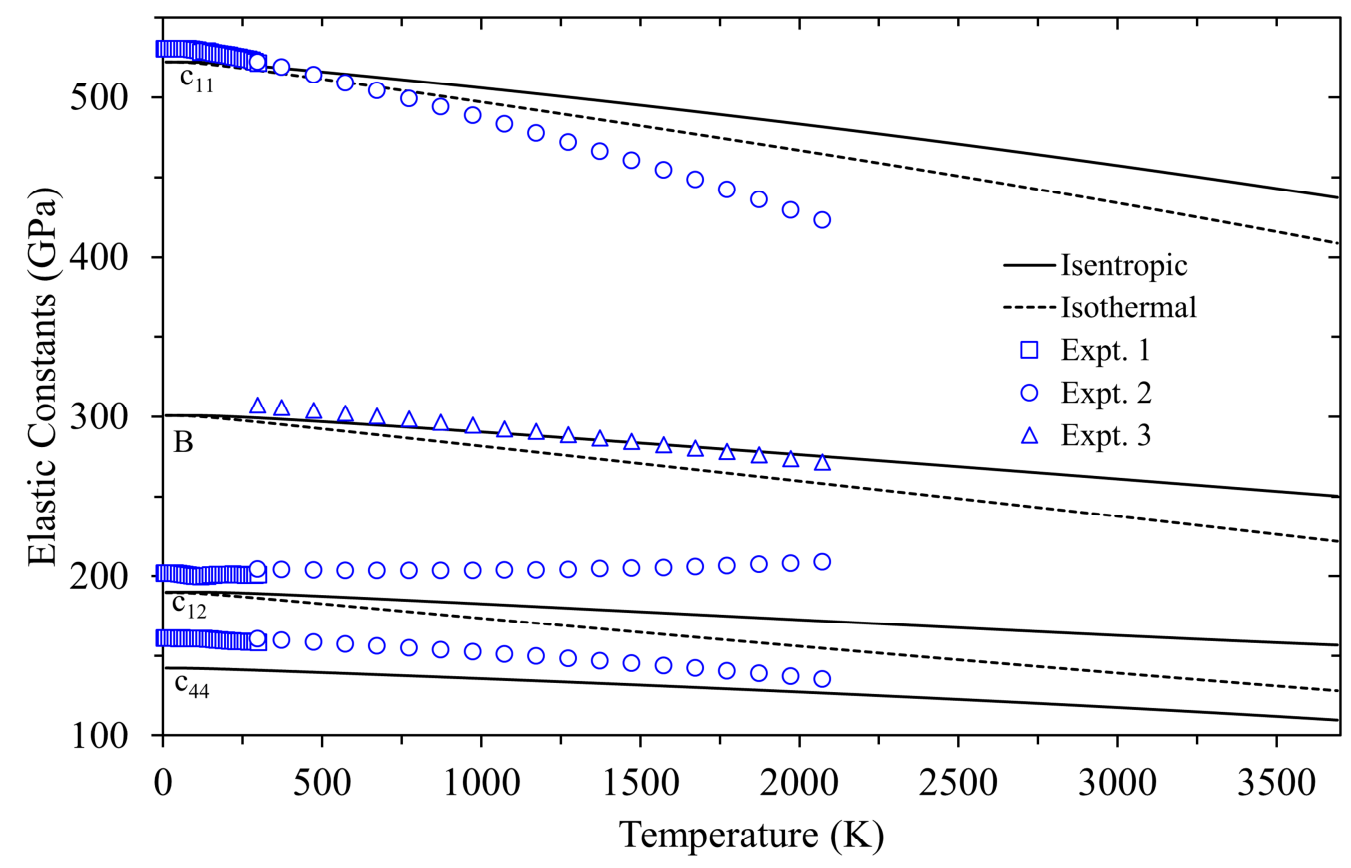




\section{Reference:}

[1] E. Lassner, W.D. Schubert, Tungsten: properties, chemistry, technology of the elements, alloys, and chemical compounds, Springer, 1999.

[2] P. Fink, J. Miller, D. Konitzer, Rhenium reduction-alloy design using an economically strategic element, JOM. 62 (2010) 55-57.

[3] W.D. Klopp, Review of ductilizing of group VIA elements by rhenium and other solutes, DTIC Document, 1968.

[4] P.L. Raffo, Yielding and fracture in tungsten and tungsten-rhenium alloys, J. Less Common Met. 17 (1969) 133-149.

[5] S.L. Shang, A. Saengdeejing, Z.G. Mei, D.E. Kim, H. Zhang, S. Ganeshan, Y. Wang, Z.K. Liu, First-principles calculations of pure elements: Equations of state and elastic stiffness constants, Comput. Mater. Sci. 48 (2010) 813-826.

[6] X.L. Liu, B.K. VanLeeuwen, S.L. Shang, Y. Du, Z.K. Liu, On the scaling factor in Debye-Grüneisen model: A case study of the $\mathrm{Mg}-\mathrm{Zn}$ binary system, Comput. Mater. Sci. 98 (2015) 34-41.

[7] X.Q. Chen, H. Niu, D. Li, Y. Li, Modeling hardness of polycrystalline materials and bulk metallic glasses, Intermetallics. 19 (2011) 1275-1281.

[8] D. Kim, S.L. Shang, Z.K. Liu, Effects of alloying elements on elastic properties of Ni by first-principles calculations, Comput. Mater. Sci. 47 (2009) 254-260.

[9] J.R. Rice, R. Thomson, Ductile versus brittle behaviour of crystals, Phil. Mag. 29 (1974) 73-97.

[10] Z. Guo, A.P. Miodownik, N. Saunders, J.P. Schillé, Influence of stacking-fault energy on high temperature creep of alpha titanium alloys, Scr. Mater. 54 (2006) 2175-2178.

[11] S.L. Shang, D.E. Kim, C.L. Zacherl, Y. Wang, Y. Du, Z.K. Liu, Effects of alloying elements and temperature on the elastic properties of dilute Ni-base superalloys from firstprinciples calculations, J. Appl. Phys. 112 (2012). doi:10.1063/1.4749406.

[12] S. Ganeshan, S.L. Shang, Y. Wang, Z.K. Liu, Effect of alloying elements on the elastic properties of Mg from first-principles calculations, Acta Mater. 57 (2009) 3876-3884.

[13] G.D. Samolyuk, Y.N. Osetsky, R.E. Stoller, The influence of transition metal solutes on the dislocation core structure and values of the Peierls stress and barrier in tungsten., J. Phys. Condens. Matter. 25 (2013) 025403. 
[14] G. Kresse, J. Furthmueller, Efficient iterative schemes for ab initio total-energy calculations using a plane-wave basis set, Phys. Rev. B. 54 (1996) 11169.

[15] G. Kresse, D. Joubert, From ultrasoft pseudopotentials to the projector augmented-wave method, Phys. Rev. B. 59 (1999) 1758..

[16] J.P. Perdew, Y. Wang, Accurate and simple analytic representation of the electron-gas correlation energy, Phys. Rev. B. 45 (1992) 13244.

[17] M. Methfessel, A.T. Paxton, High-Precision Sampling for Brillouin-Zone Integration in Metals, Phys. Rev. B. 40 (1989) 3616-3621.

[18] P.E. Bloechl, Projector augmented-wave method, Phys. Rev. B. 50 (1994) 17953.

[19] K. Momma, F. Izumi, VESTA: a three-dimensional visualization system for electronic and structural analysis, J. Appl. Crystallogr. 41 (2008) 653-658.

[20] K. Momma, F. Izumi, VESTA 3 for three-dimensional visualization of crystal, volumetric and morphology data, J. Appl. Crystallogr. 44 (2011) 1272-1276.

[21] S. Shang, Y. Wang, Z.-K. Liu, First-principles elastic constants of $\alpha$ - and $\theta-\mathrm{Al}_{2} \mathrm{O}_{3}, \mathrm{Appl}$. Phys. Lett. 90 (2007)

[22] G. Simmons, H. Wang, Single crystal elastic constants and calculated aggregate properties: a handbook, M.I.T. Press, 1971.

[23] D.C. Chrzan, M.P. Sherburne, Y. Hanlumyuang, T. Li, J.W. Morris, Spreading of dislocation cores in elastically anisotropic body-centered-cubic materials: The case of gum metal, Phys. Rev. B - Condens. Matter Mater. Phys. 82 (2010) 1-5.

[24] Y. Wang, J.J. Wang, H. Zhang, V.R. Manga, S.L. Shang, L.Q. Chen, Z.K. Liu, A firstprinciples approach to finite temperature elastic constants., J. Phys. Condens. Matter. 22 (2010) 225404.

[25] S.L. Shang, Y. Wang, D. Kim, Z.K. Liu, First-principles thermodynamics from phonon and Debye model: Application to $\mathrm{Ni}$ and $\mathrm{Ni}_{3} \mathrm{Al}$, Comput. Mater. Sci. 47 (2010) 10401048 .

[26] Y. Wang, Z.K. Liu, L.Q. Chen, Thermodynamic properties of $\mathrm{Al}, \mathrm{Ni}, \mathrm{NiAl}$, and $\mathrm{Ni}_{3} \mathrm{Al}$ from first-principles calculations, Acta Mater. 52 (2004) 2665-2671.

[27] G.F. Davies, Effective elastic moduli under hydrostatic stress-I. quasi-harmonic theory, J. Phys. Chem. Solids. 35 (1974) 1513-1520.

[28] J.H. Stathis, D.I. Bolef, Elastic constants of tungsten between 4.2 and 77 K, J. Appl. Phys. $51(1980) 4770$. 
[29] H.W. King, Quantitative size-factors for metallic solid solutions, J. Mater. Sci. 1 (1966) 79-90.

[30] T. Wang, L.Q. Chen, Z.K. Liu, Lattice parameters and local lattice distortions in fcc-Ni solutions, Metall. Mater. Trans. A. 38 (2007) 562-569.

[31] M. Garfinkle, Effect of rhenium alloying on lattice dilation of the group VI-a refractory metals, Metall. Mater. Trans. B. 1 (1970) 1062-1063.

[32] W.B. Pearson, A handbook of lattice spacings and structures of metals and alloys, 1967.

[33] A.R. Miedema, F.R. De Boer, P.F. De Chatel, Empirical description of the role of electronegativity in alloy formation, J. Phys. F Met. Phys. 3 (1973) 1558.

[34] Li, P. Wu, Correlation of bulk modulus and the constituent element properties of binary intermetallic compounds, Chem. Mater. 13 (2001) 4642-4648.

[35] J.H. Rose, H.B. Shore, Uniform electron gas for transition metals: Input parameters, Phys. Rev. B. 48 (1993) 18254.

[36] S. Shang, Y. Wang, R. Arroyave, Z.K. Liu, Phase stability in $\alpha$ - and $\beta$-rhombohedral boron, Phys. Rev. B. 75 (2007) 92101.

[37] K. Edalati, T. Furuta, T. Daio, S. Kuramoto, Z. Horita, High strength and high uniform ductility in a severely deformed iron alloy by lattice softening and multimodal-structure formation, Mater. Res. Lett. 3 (2015) 197-202.

[38] S. Kuramoto, T. Furuta, N. Nagasako, Z. Horita, Lattice softening for producing ultrahigh strength of iron base nanocrystalline alloy, Appl. Phys. Lett. 95 (2009) 3.

[39] A.S. Argon, Strengthening mechanisms in crystal plasticity, Oxford University Press Oxford, 2008.

[40] J.R. Stephens, W.R. Witzke, Alloy softening in group VIA metals alloyed with rhenium, J. Less Common Met. 23 (1971) 325-342.

[41] R. Lowrie, A.M. Gonas, Dynamic elastic properties of polycrystalline tungsten, 24-1800 C, J. Appl. Phys. 36 (1965) 2189-2192. 